\title{
Improving Reliability of Distribution System by Optimum Placement of DGs
}

\author{
Tanaya D. Gulhane ${ }^{1}$, Prof. Ganesh Mhatre ${ }^{2}$ \\ Electrical Engineering Department, Yadavrao Tasgaonkar Institute of Engg, and Technology, Karjat, India ${ }^{1}$ \\ Asst. Professor, Electrical Engineering Department, NHITM College, Thane, India ${ }^{2}$
}

\begin{abstract}
In the emerging power systems Distributed Generators (DGs) are expected to play a vital role as it has a great impact on the operation and planning of distribution network. Installing the DG at optimum location enhances the system performance and improves the reliability. In this paper a method is proposed for finding the optimal location of DGs with their optimum numbers and a goal to maximize reliability of system, reduce the total power loss while improving the voltage profile of the system for different load conditions using Particle Swarm Optimization (PSO) and Neural Network (NN) to fix DGs and evaluate the amount of power to be generated.
\end{abstract}

Keywords: Optimal Location, Power Loss, DG, PSO, Optimal Size, Neural Network.

\section{INTRODUCTION}

Utilities design and plan successively the expansion of their electrical networks in order to meet the load growth and to properly supply their consumers by construction of new substations or the expansion of those already exists. However, companies began to appraise new manners of expanding their capacities simulating the addition of new power sources, Distributed Generation (DG) to the system. Distributed generation units is emerging massively in the electric power system infrastructure and growing market in recent years. It is small-scale generating power facility installed in the distribution system, value typically less than $10 \mathrm{MW}$. It is a technique that reduces the power loss in transmission lines as the required generation of energy is closer to the load center and places of consumption. The distributed generation, also termed as embedded generation or dispersed generation or decentralized generation, has been defined as electric power source connected directly to the distribution network or on the customer site of the meter [1].

DGs can provide effective solution to solve the problem of saturated existing system due to demand growth. They are particularly located and operated to minimize the system power losses, reinforce grid and improve system reliability and efficiency. Some of the major advantages while installing DG units in distribution level are

- Peak load saving,

- Upgrading the reliability,

- Voltage stability improvement,

- Decreasing power losses,

- Power quality enhancement,

- Less polluting emissions [2]

- Improved security

- Grid strengthening

- Reduced on-peak operating cost
The advantageous effects of DG mainly depend on its location and size. DG installation in the power grid requires careful considerations for factors such as stability, reliability, protection coordination, power loss and power quality issues, etc. The setting up of a DG unit at a nonoptimal place and improper sizing will consequence in increased system losses and increased operational cost, poor reliability and power quality. This issue is a necessary process to maintain the stability and reliability of existing system effectively before DG is connected to a power grid. Therefore, the optimal choice of DG location and sizing has a countless effect on a system performance with regards to power loss, voltage profile, system stability and reliability.

A lot of technologies are used for DG sources such as photo voltaic cells, wind generation, combustion engines, and fuel cells and there are some other types of generation from the natural or artificial resources that are available in the geographical area [3]. Usually, DGs are attached with the already existing distribution system and lot of studies are performed to find out the best location and size of DGs to produce highest benefits [4]. The effect of placing a DG on network indices will be different based upon its type and location and (predict) load at the connection point [5]. There are multiple probable benefits of DG systems both to the consumer and the electric supply provider that allow for greater electrical flexibility along with energy security.

This paper presents a technique to identify optimal location of DG \& power generated by them using PSO and Neural Network. The paper is organized in following manner: section II is brief review of previous related work, intelligent approach for optimal DG placement is presented in section III and section IV concludes the paper. 
Vol. 5, Issue 1, January 2017

\section{REVIEW OF RELATED WORK}

Some of the research related to optimal placement and its size with reliability of distributed generator is as trails.

In [6] Amanifar et al. have proposed a PSO algorithm that finds out the optimal placement and size of DGs which was performed on a 15- bus test system and objectives was to reduce total cost of the system, real power loss and the number of DGs to be connected. The result has indicated that the PSO have the capability to search the best position and size of DGs on power system network. Injeti et al. [7] presents a method for optimal planning and operation of DG unit placement and sizing using a fuzzy logic and an analytical method respectively. Mohammadi et al. [8] have proposed an optimal DG unit placement using Genetic Algorithm (GA) containing a set of rules which is used to decide the DG unit position with highest suitability index. Simulation results have shown good reduction not only in power loss but also in enhancing system reliability.

A method using Fuzzy Logic and PSO technique was suggested to install DG in the radial distribution systems by Lalitha et al. [9] to reduce the real power losses and to make some enhancements in the voltage profile. A twostage methodology has been proposed where in the initial stage, the best DG location was established using fuzzy approach, and in the succeeding stage, the size of the DGs is set using PSO for extreme loss reduction.

Paliwal et al. [10] have explored the different influences of DG unit's installation on various functions such as, electric power losses, reliability and voltage profile of distribution networks. The study aims to find optimal distributed generation position for loss reduction subjected to constraint of voltage regulation in distribution network. Further it was examined for different increased levels of Reliability.

\section{INTELLIGENT APPROACH FOR OPTIMAL DG PLACEMENT}

Distributed generators are frequently used in modern distribution system with an objective to upsurge the power flow in the system and also to attain some improvements in the reliability and decrease the total power losses of the system. Assigning suitable position of DG units on the buses of network with its generated power depending on load demand increases the quality of power of the system. By considering the shortcomings of identification of the optimal location to fix DG and the amount of power to be generated by the DG, a hybrid system is proposed which consist of PSO and neural network to increase the reliability in distribution systems by connecting DGs. The method be made of three stages: in the initial stage training dataset is created using Particle Swarm Optimization for the number of DGs to be connected; in the middle stage Neural Network is used to categorize the finest location for the given number of DGs \& in the final stage Particle Swarm Optimization is used to calculate the amount of power to be produced by the DGs to reduce the power losses. Figure 1 depicts overall process.

Power flow between the buses is to be calculated before identification of fixing DG in the system where it is computed using Newton Raphson (NR) method. It is a powerful method of solving non-linear algebraic equations. It is indeed the practical method of load flow solution of large power networks as it works faster and converges in most cases as compared to other methods. Equation (1) and equation (2) calculates the real $\left(\mathrm{P}_{\mathrm{i}}\right)$ and reactive $\left(\mathrm{Q}_{\mathrm{i}}\right)$ power between the buses

$$
\begin{gathered}
P_{i}=\sum_{k=1}^{N} V_{i} \times V_{k}\left(X_{i k} \times \cos \theta_{i k}+Y_{i k} \times \sin \theta_{i k}\right) \\
Q_{i}=\sum_{k=1}^{N} V_{i} \times V_{k}\left(X_{i k}\right. \\
\left.\quad \times \sin \theta_{i k}-Y_{i k} \times \cos \theta_{i k}\right)
\end{gathered}
$$

Where, $\mathrm{V}_{\mathrm{i}}$ and $\mathrm{V}_{\mathrm{k}}$ are voltages at $\mathrm{i}$ and $\mathrm{k}$ bus respectively, $\mathrm{N}$ is total number of buses. $\theta_{i k}$ is the angle between $\mathrm{i}$ and $\mathrm{k}$ bus. $X_{i k}$ and $Y_{i k}$ are conductance \& susceptance values respectively. There are two important factors which influence real and reactive powers are voltage and angle values. After calculating the power flow, further optimal location for fitting the DGs in the system is determined which is computed by NN. For training the NN dataset is required that is further generated using PSO to achieve optimal locations. The procedure that takes place in the suggested method comprises of: (i) Generating training dataset using PSO (ii) Neural-network to identify optimal location of DGs (iii) Computing power to be generated by DG. This process is discussed in the following sections

\section{A. Initializing Training Dataset using PSO:}

The several possible optimal posts of DGs in the system is identified using Particle Swarm Optimization which consists of four stages namely, generating initial particle, evaluation function, updating initial particle and termination. The training dataset is generated by PSO for possible connection for fixing one, two, three and four DGs in the system and the finest set of combinations are carefully chosen on the basis of total power losses in the system.

1) Generating initial particle: The first step in PSO is creating initial particle to develop training dataset. In this, the particles depend on number of distributed generators to be attached in modern system. If number of distributed generators to be fixed is $\mathrm{z}$, then number of particles is three times z. The output particle comprises DG position, $P_{i}$ and $Q_{i}$, where $P_{i} \& Q_{i}$ has values between its minimum and maximum values.

2) Evaluation function: This function is used in PSO to recognize the best particle from the set of initialized particle. The total power loss $\left(\mathrm{P}_{\text {loss }}\right)$ represents evaluation function in this method which is given as equation (3). 
Vol. 5, Issue 1, January 2017

$$
P_{\text {loss }}=\sum_{i, j=1}^{N} \operatorname{Real}\left[\operatorname{Conj}\left(\left(V_{m}(i)\right) \times\left(V_{m}(j)\right)\right) \times Y_{i j}\right.
$$

Where $Y_{i j}$ is $Y$-bus matrix and $B$ is the base MVA, $V_{m}$ is voltage magnitude. Using this $\mathrm{P}_{\text {loss }}$ optimal location of $\mathrm{DG}$ is fixed.

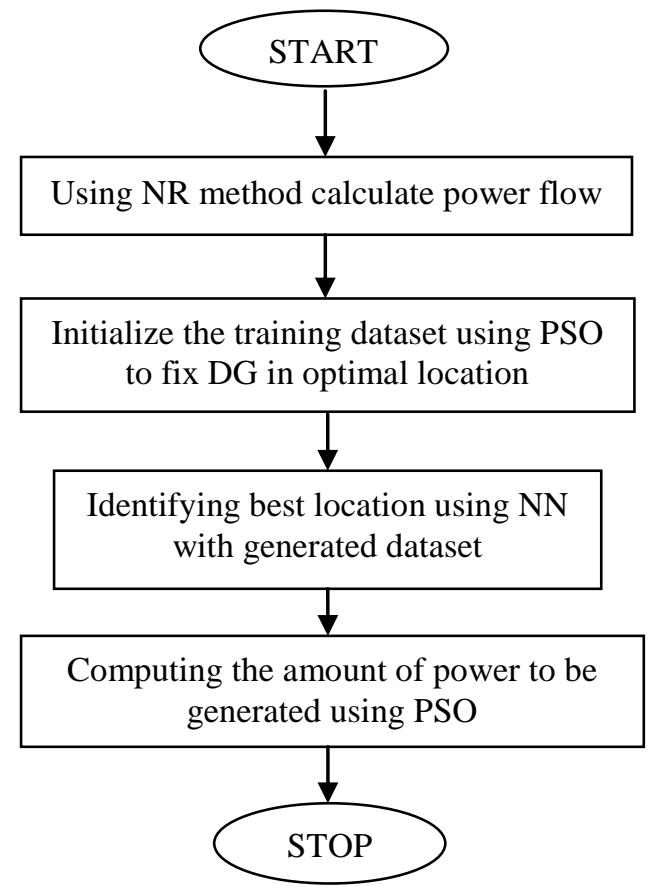

Fig. 1: Procedure of method projected

3) Updating initial particle: In PSO updating the diverse particles in the system is an essential process. In this stage, the initial particles produced are updated and the evaluation function is repeated. The particles velocity are updated using the equation (4) and particle position is updated using equation (5).

$$
\begin{aligned}
& \mathrm{v}[]=\mathrm{v}[]+\mathrm{c} 1 * \operatorname{rand}(\mathrm{)} *(\operatorname{pbest}[]-\operatorname{present}[])+\mathrm{c} 2 * \\
& \operatorname{rand}() *(\operatorname{gbest}[]-\operatorname{present}[]) \\
& \operatorname{present}[]=\operatorname{present}[]+\mathrm{v}[]
\end{aligned}
$$

where $v[$ ] is the particle velocity, present [ ] is the current particle, pbest[] and gbest[ ] are the best fitness values and best values for any particle in the population respectively, rand ( ) is the random number between $(0,1)$ and $c 1, c 2$ are learning factors.

4) Termination: The above procedure is repeated, until best particle criterion is met, usually a sufficiently good fitness value or a maximum number of generations. After completion of termination process, a probable set of location for fixing DGs and their corresponding real and reactive power values are obtained. The above process is recurrent for different number of DGs connected in the system and this probable set is considered as dataset from which the best set is selected by neural network

B. Identification of Optimal Location using $\mathrm{NN}$ :

For identification of the optimal placement of DGs the Neural-network is used. $\mathrm{NN}$ is used to select the best location from the few locations computed in the first stage, which satisfy the active and reactive power constraints. Usually, Neural-network consists of two stages:

1) Training stage: The network is accomplished based on the training dataset.

2) Testing stage: If the number of DGs to be connected is given as input, it gives the best place of installation of DGs in the system.

Its training have 3 layers - input layer, hidden layer and output layer. In the described method, input layer be made of $\mathrm{p}$ layers, hidden layers be made of q layers and output layers be made of $3 p$ layers. The configuration of neural network used here is as shown [11] in figure 2. The neural network is trained using the generated dataset.

The steps for training the Neural-network are:

1) Initialize the input weight of each neuron

2) Apply the training dataset to the network. Here, p is the input to the network and $D_{1}, D_{2} \ldots D_{3 p}$ are the outputs of the network. Equation (6), Equation (7) and Equation (8) signify the activation functions performed in the input and output layers.

3) Adjust the weights of all neurons.

4) Determine the optimal placement of DGs to be connected.

$$
\begin{aligned}
& D_{1}=\sum_{r=1}^{n} A_{2 r 1} Y_{1}(r) \\
& D_{2}=\sum_{r=1}^{n} A_{2 r 2} Y_{2}(r) \\
& D_{3 p}=\sum_{r=1}^{n} A_{2 r(3 p)} Y_{(3 p)}(r)
\end{aligned}
$$

Where

$$
Y(r)=\frac{1}{1+\exp \left(-A_{11} \cdot p\right)}
$$

In the next (testing) stage, if input is considered as the number of DGs to be connected in the system, then best locations to fix the DGs will be given as output by the neural network and it will also reduce the power loss to improve the reliability of the system. After obtaining the optimal location for DGs, the succeeding step is to figure the amount of power to be generated by DGs with improved reliability which is computed using PSO. 
IJIREEICE

Vol. 5, Issue 1, January 2017

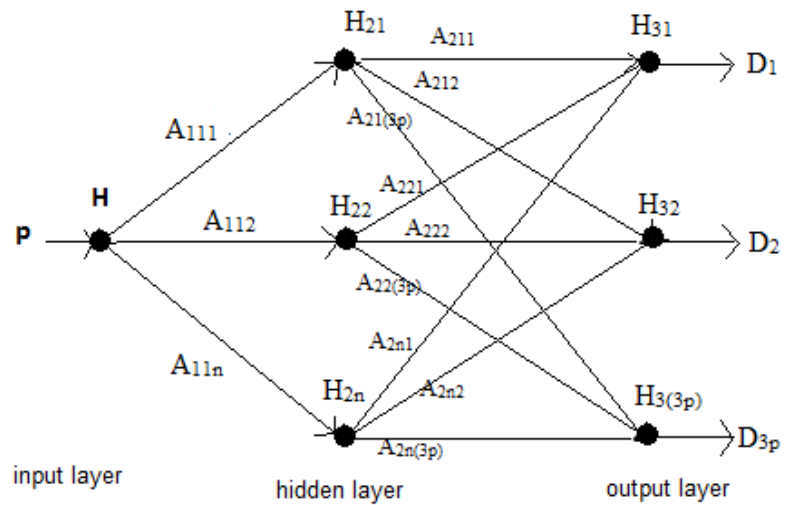

Fig. 2: Neural Network

C. Computation of Power using PSO:

The amount of power to be produced by each generator is computed using PSO in this stage, after obtaining optimal location of DGs in the second stage. The first step in PSO is to generate initial particle where the real and reactive power are the two input particles and must satisfy the constraints i.e. their values must be less than maximum limit and more than minimum limit. After the generation of initial particle, further step in PSO is evaluation function. This function considered is represented in equation (3). The reliability parameters taken here are expected energy not supplied (EENS) and system expected outage cost (ECOST). They are calculated using equation (10) and equation (11) respectively.

$$
\begin{aligned}
& \text { EENS }=\sum_{\mathrm{i}} \sum_{\mathrm{k}} \mathrm{L}_{\mathrm{k}} \mathrm{h}_{\mathrm{i}} \mathrm{t}_{\mathrm{i}} \\
& \mathrm{ECOST}=\sum_{\mathrm{i}} \sum_{\mathrm{k}} \mathrm{L}_{\mathrm{k}} \mathrm{h}_{\mathrm{i}} \mathrm{C}_{\mathrm{ik}}\left(\mathrm{t}_{\mathrm{i}}\right)
\end{aligned}
$$

Where, $t_{i}$ is the interruption time, $L_{k}$ is the load, $h_{i}$ is failure rate and $\mathrm{C}_{\mathrm{ik}}$ is customer interruption cost. The next step is updating initial particle which is same as described in section A.3. In the last step of PSO i.e. termination operation, the best particle here DG with less power loss and with an improved reliability is selected as the best power to be generated by DG.

\section{CONCLUSION}

This paper has presented overall procedure of projected method to improve the reliability of system, reduce the power losses, voltage profile and power quality using intelligent techniques. The techniques employed to state the issue of Distributed generation siting and sizing used here are Particle Swarm Optimization and Neural Network. Testing this on IEEE bus system will prove that reliability has enhanced along with the power quality of modern system. The reliability indices EENS and ECOST values will show the reduction in their values. The two methods amalgamation can effectively detect the DG location with its suitable size and their numbers at optimal location to minimize the total power loss and show considerable improvement in reliability. Based upon this it is resolved that heuristic approaches are more feasible and modest solution techniques than the conservative ones. A hybrid approach of two or more ways as depicted here contribute a better option by integrating the benefits and discarding the shortcomings.

\section{ACKNOWLEDGEMENT}

Authors are thankful to the HOD and Department of Electrical Engineering, Y.T.I.E.T., Karjat, Mumbai for their kind academic support.

\section{REFERENCES}

[1] Mansooreh Zangiabadi, Rene Feuillet and Hamid Lesani, "An Approach to Deterministic and Stochastic Evaluation of the Uncertainties in Distributed Generation Systems", In Proceedings of 20th International Conference on Electricity Distribution, pp. 14, Prague, 2009.

[2] Priyantha D.C. Wijayatunga, W. J. L. S. Fernando and Ram M. Shrestha, "Impact of distributed and independent power generation on greenhouse gas and emissions:Sri Lanka", Energy conservation and management, 17 March 2004

[3] K. Manjunatha Sharma and K. P. Vittal, "A Heuristic Approach to Distributed Generation Source Allocation for Electrical Power Distribution Systems", Iranian Journal of Electrical \& Electronic Engineering, Vol. 6, No. 4, pp. 224-231, Dec. 2010.

[4] Deependra Singh, Devender Singh and K S Verma, "Distributed Generation Planning Strategy with Load Models in Radial Distribution System", International Journal of Computer and Electrical Engineering, Vol. I, No. 3, pp. 362-375, Aug 2009.

[5] Amin Hajizadeh, Ehsan Hajizadeh, "PSO-Based Planning of Distribution Systems with Distributed Generations", International Journal of Electrical and Electronics Engineering, Vol. 2, No. I, pp. 33-38, 2008.

[6] O. Amanifar and M.E. Hamedani Golshan, "Optimal Distributed Generation Placement and Sizing for Loss and THD Reduction and Voltage Profile Improvement in Distribution Systems using Particle Swarm Optimization and Sensitivity Analysis", International Journal on Technical and Physical Problems of Engineering, Vol. 3, No. 2, pp. 47-53,2011.

[7] S. Kumar Injeti and Navuri P Kumar, "Optimal Planning of Distributed Generation for Improved Voltage Stability and Loss Reduction", International Journal of Computer Applications, Vol. 15, No. 1, pp. 40-46, Feb 2011.

[8] Mohammad Mohammadi and M. Akbari Nasab, "DG Placement with Considering Reliability Improvement and Power Loss Reduction with GA Method", Research Journal of Applied Sciences, Engineering and Technology 3(8): 838-842,2011.

[9] M . Padma Lalitha, V. c. Veera Reddy, N. Sivarami Reddy and K. Sivagangadhar Reddy, "Clonal Selection Algorithm for DG Sources Allocation for Minimum Loss in Distribution System", International Journal of Computer Science \& Emerging Technologies, Vol. I, pp. 85-91, Oct 2010.

[10] Priyanka Paliwal and N. P. Patidar, "Distributed Generator Placement for Loss Reduction and Improvement in Reliability", World Academy of Science, Engineering and Technology, Vol. 69, pp. 809-813, 2010.

[11] S Chandrashekhar Reddy, P.V.N. Prasad and A. Jaya Laxmi, "Reliability Improvement of Distribution System by Optimal Placement of Distributed Generator Using Genetic Algorithm and Neural Network", Journal of Electrical Engineering

[12] S Chandrashekhar Reddy, P.V.N. Prasad and A. Jaya Laxmi, "Power Quality and Reliability Improvement of Distribution System by Optimal Number, Location and Size of DGs Using Particle Swarm Optimization”, 978-1-4673-2605-6/12/2012 IEEE. 